ISSN 1991-8631

\title{
Les produits et sous-produits du bananier dans l'alimentation animale
}

\author{
Kouamé Guy Marcel BOUAFOU ${ }^{1 *}$, Brou André KONAN ${ }^{2}$, Koffi Gabouet KOUAME ${ }^{2}$ \\ et Séraphin KATI-COULIBALLY ${ }^{2}$ \\ ${ }^{1}$ Section des Sciences de la Vie et de la Terre, Département des Sciences et Technologie, Ecole Normale \\ Supérieure d'Abidjan (Côte d'Ivoire), 25 BP 663 Abidjan 25, Tel : +22566529238/+22540309965 \\ ${ }^{2}$ Laboratoire de Nutrition et Pharmacologie, UFR-Biosciences, Université de Cocody (Côte d'Ivoire), 22 \\ B.P. 582 Abidjan 22. \\ *Auteur correspondant, E-mail : publicationbouafou@yahoo.fr
}

\section{RESUME}

L'objectif de ce travail est de montrer que les produits et sous-produits du bananier peuvent être incorporés dans l'alimentation animale sans risque. Ces produits et sous-produits agricoles ont été plusieurs fois utilisés dans l'alimentation des ruminants, du porc, du lapin et de la volaille sous forme ensilée, farineuse ou hachée à des taux variables. Les résultats obtenus sont comparables à ceux obtenus avec des aliments couramment utilisés en élevage. Les feuilles du bananier, le son pseudo-tronc, la banane et ses déchets peuvent servir dans l'alimentation animale.

(c) 2012 International Formulae Group. All rights reserved.

Mots clés : Bananier, banane, sous- produits, alimentation.

\section{INTRODUCTION}

En Afrique et singulièrement en Côte d'Ivoire, les fermiers sont confrontés aux coûts élevés des intrants alimentaires dans leur activité. Par exemple, les coûts du kilogramme du tourteau de soja ou de la farine de maïs varient de170 à 325 F CFA (Bouafou, 2007). Ces constats commandent d'envisager des solutions alternatives: remplacer partiellement ou totalement les intrants alimentaires classiques, par d'autres sources disponibles et moins chers. Dans cette donne, Mohapatra et al. (2010) indique que les produits et sous- produits du bananier (Musa cavendishi, Musa paradisia, Musa sapientum) sont exploitables dans l'alimentation. Et pourtant, l'emploi des produits et sous- produits du bananier dans l'élevage n'est pas encore une pratique commune et singulièrement en Côte d'Ivoire, grande productrice africaine de bananes.

\section{CONSERVATION DES PRODUITS ET SOUS-PRODUITS DU BANANIER}

La meilleure manière de conserver la banane verte est l'ensilage et ce pour des raisons nutritionnelles qu'économiques. De cette manière, la banane verte garde sa caractéristique essentielle qui est sa richesse en amidon de haute valeur énergétique (Le Dividich et al., 1976).

Sur le plan pratique, l'ensilage de la banane mure présente l'avantage de ne pas nécessiter de broyage (Le Dividich et al., 
1976). Lorsqu'on ensile la banane mure (mûrissement artificielle à l'acétylène) les pertes en matières sèches en un mois sont considérables $(37 \%)$; elle tombe à $16 \%$ avec la banane verte broyée (Sève et al., 1972).

L'ensilage des feuilles de banane avec d'autres ingrédients peut être une stratégie intéressante pour alimentation animale. Les feuilles de banane $(75 \%)$ et la paille de blé $(25 \%)$ ensilées avec de la mélasse et l'urée pourraient être utilisées dans l'alimentation des vaches (Baloch et al., 1988).

Pour l'alimentation des moutons, l'ensilage des feuilles de bananier avec l'urée est préférable (Shoukry, 1999). Les feuilles de banane ensilées $(40 \%)$ avec de la litière de poulets de chair $(60 \%)$ et de la mélasse ou du lactosérum peuvent être introduites dans l'alimentation des buffles en lactation (Khattab et al., 2000).

Un bon ensilage peut être obtenu à partir de $50 \%$ de bananes vertes et $50 \%$ d'herbes hachées ou à partir de bananes vertes hachées associée à 1,5\% de mélasse (Thom pson, 1995).

\section{COMPOSITIONS CHIMIQUES DES PRODUITS ET SOUS- PRODUITS DU BANANIER}

Les feuilles du bananier contiennent environ $85 \%$ d'eau et une teneur en protéine comprise entre 10 et $17 \%$ de matière sèche (Ffoulkes et al., 1977). Les pseudo-troncs du bananier contiennent surtout de l'eau, 92 à $95 \%$, très peu de protéines (3- 4,5\% de matière sèche) (Ffoulkes et al., 1977). Ils ont une teneur élevée en fibre: 50 à $70 \%$ de matière sèche (Heuzé et al., 2011).

La banane est hautement nutritive et plus facile à digérer que plusieurs autres fruits. Elle est riche en potassium, en calcium mais pauvre en sodium (Adisa and Okey, 1987; Wall, 2006). Les compositions chimiques de la pulpe et de l'épluchure de la banane douce sont consignées dans le Tableau 1.

Le Tableau 2 présente les compositions chimiques des produits et sous- produits du bananier plantain. Les déchets de banane constituent un produit riches en eau et en minéraux, mais très pauvres en azote (Cordeiro et al., 2004 ; Oliviera et al., 2004). Les déchets de banane désignent ici essentiellement les épluchures de banane. Ils sont exclusivement énergétiques. Leur broyage et surtout leur cuisson améliore leur valeur alimentaire et principalement leur digestibilité (Le Dividich and Canope, 1972).

La pulpe de banane, en raison de sa valeur nutritive peut constituer un excellent aliment pour le serpent (Ruales et al., 1990).

\section{INCORPORATION DES PRODUITS ET SOUS- PRODUITS DU BANANIER DANS L'ALIMENTATION ANIMALE \\ Epluchure (peau) de banane \\ Chezle porc}

Les déchets de banane peuvent constituer en pays tropical (Guadeloupe) un aliment pour le porc en croissance et représentent jusqu'à 55\% de matière sèche ingérée (Le Dividich et Canope, 1972).

\section{Chez les bovins et la volaille}

Chez les ruminants, une étude menée par Abiliza et Muya (1991), en Tanzanie, révèle que les feuilles, les épluchures (peaux) de banane et les pseudo-troncs du bananier possèdent une certaine valeur nutritive pour être un bon aliment pour les vaches laitières et les bovins.

En outre, ils sont souvent exploités pour l'alimentation du bétail et la volaille (7,5\%) (Dormond et al., 1998 ; Emaga et al., 2008 ; Adéniji et al., 2008).

\section{Chez les lapins}

Les éleveurs ont encouragé à intégrer les épluchures de plantain à hauteur de $75 \%$ en tant que substitut du maïs dans l'alimentation des lapins. Ce qui permet de réduire le coût total des aliments qui constitue plus de $60 \%$ des charges de production (Ajasin et al., 2006).

Omole et al. (2008) ont remplacé dans le régime du lapin sevré, la fraction de farine de maïs par la farine d'épluchures (peaux) séchées de banane plantain à différents taux : 
$25 \%, 50 \%, 75 \%$ et $100 \%$. Les résultats ont montré qu'il n'y avait pas de différences significatives $(\mathrm{p}<0,05)$ entre les gains de poids et l'indice de conversion alimentaire issues du régime témoin (à base de farine de maï) et le régime contenant $75 \%$ de farine d'épluchures séchées de banane plantain. Ils ont conclu que la farine d'épluchures séchées de banane plantain pouvait remplacer la farine de maïs à hauteur de $75 \%$ dans le régime du lapin sevré.

L'effet des épluchures de plantain mûr sur la performance de croissance, les caractéristiques de la carcasse, le développement des muscles et des coûtsavantages a été étudié chez des lapins sevrés. En effet, l'inclusion d'épluchures de plantain dans des régimes de lapins n'a pas eu d'effet néfaste sur les animaux (Ogunsipe and Agbede, 2010).

\section{Pulpe de banane \\ Valeur nutritionnelle}

La valeur nutritionnelle de 1 a pulpe de banane a été étudiée. Ainsi, la digestibilité de la matière sèche est plus faible avec les bananes vertes ensilées $(80,6)$ ou mures $(77,3)$ qu'avec la banane fraîche $(83,4)$. La digestibilité des matières azotées, déjà faible avec la banane verte ensilée $(72,2)$ tombent à 68,4 avec la banane verte ensilée et à 64,2 avec la banane mure ensilée (Sève et al., 1972). Les coefficients d'utilisation digestive apparente de la matière sèche, de la matière organique et des protéines d'un régime à base de manioc sont supérieurs à ceux d'un régime à base de banane (Le Dividich et Canope, 1974). La digestibilité apparente de la matière sèche d'un régime à base de banane fraîche est significativement supérieure $(p>0,05)$ à celle des régimes à base d'ensilage et il en est de même pour la digestibilité des matières organiques (Sève et al., 1976). Par contre, la digestibilité apparente d'azote des régimes à base de banane ensilée ou non, est significativement inférieure $(\mathrm{p}>0,05)$ à celle d'un aliment complet du commerce. En revanche, le coefficient de rétention protéique n'est pas modifiée par la présence de banane dans la ration, bien que l'ensilage, surtout celui de banane mure, tende à le diminuer (Sève et al., 1976).

L'efficacité alimentaire des régimes à base de banane est inférieure $(p>0,05)$ à celle d'un aliment complet du commerce (Sève et al., 1976). L'efficacité alimentaire des régimes à base de banane et de manioc est la même. Elle augmente de $10 \%$ lorsque le taux de protéines s'accroît (Le Dividich and Canope, 1974).

\section{Chezle porc}

L'emploi de la banane dans l'alimentation des porcs a donné des résultats probants. Le coefficient d'efficacité protidique d'un régime à base de banane chez le porcelet sevré a tendance à diminuer légèrement lorsque le taux de protéines augmente (Le Dividich and Canope, 1974).

La farine de pulpe de banane verte incorporée à un taux de $50 \%$ à la ration de sevrage du porcelet âgé de 5 à 9 semaines leur permet de réaliser des performances de croissance comparables à celles obtenues avec a farine de manioc. L'utilisation digestive apparente des principaux éléments (sauf les cendres) du régime à base de banane est toutefois significativement inférieure $(\mathrm{p}<$ $0,01)$ à celle du régime renfermant la farine de manioc. Les meilleures performances sont obtenues avec une ration présentant un taux de protéines voisin de $21,5 \%$. Un taux plus faible $(19 \%)$ ne permet ni une croissance maximale ni une bonne efficacité de la ration. Les résultats d'ensemble font apparaitre que la farine de banane possède une valeur alimentaire comparable à celle de la farine de manioc pour le porcelet âgée de 5 à 9 semaines. Elle peut être incorporée sans risque jusqu'à 50\% dans son régime (Le Dividich et Canope, 1974). Clavijo et Maner (1975) ont montré que le porc peut bien se nourrir avec la farine de banane fraîche ou sèche. Cependant, l'introduction de banane dans l'alimentation du porc en croissance-finition entraîne une vitesse de croissance limitée par rapport celle obtenue avec un aliment complet du 
commerce (Sève et al., 1976). Elle s'accompagne d'une faible digestibilité des matières azotées de la ration. En revanche, la farine de banane permet la production de carcasse de bonne qualité même si les animaux sont abattus à des poids élevés (110 $\mathrm{kg}$ ). Ainsi, la banane, aliment riche en eau peut constituer $50 \%$ de la matière sèche totale ingérée par le porc entre 30 et $90 \mathrm{~kg}$ de poids vif et $65 \%$ entre 90 et $110 \mathrm{~kg}$ (Sève et al., 1976).

Selon Sharrock (1997), la farine de banane plantain verte peut être aussi utilisée pour remplacer jusqu'à $75-80 \%$ les grains de céréales constitutifs du régime du porc.

\section{Chez le rat, la volaille et le lapin}

La banane a été également testée dans l'alimentation du rat, de la volaille et du lapin. Les performances de croissance des rats nourris avec un régime à base de farine de banane cuite ne diffèrent pas significativement $(\mathrm{p}<0,05)$ de celles de leurs congénères alimentés à base d'amidon de maïs (Cerning-Beroard et Le Dividich, 1976).

Outre le rat, la farine de banane a été utilisée pour nourrir la volaille. Mais des taux élevés dans le régime ont tendance à réduire sa croissance et l'efficacité alimentaire (Sharrock, 1997).

La valeur énergétique digestible de la banane verte est de $3640 \mathrm{bkcal} / \mathrm{kg}$ de matière sèche. Les lapins peuvent en consommer en moyenne $12 \mathrm{~g}$ par jour, soit 32\% de matière sèche ingérée (Gidenne, 1986).

\section{Feuilles de bananier}

L'emploi des feuilles du bananier dans l'alimentation animale a été le sujet de nombreux travaux. Ces travaux ont concerné les ruminants (zébu, mouton, agneaux, vache, bœuf et buffle), le lapin, le poulet et le porc.

La digestibilité des feuilles du bananier est de $65 \%$ et celles-ci sont bien consommées par le bétail. L'indice de consommation est $2,15 \mathrm{~kg}$ pour $100 \mathrm{~kg}$ de poids vif par jour (Ffoulkes et Preston, 1978).

L'incorporation de la farine de feuilles de bananier jusqu'à $40 \%$ dans la ration de fourrage a montré l'augmentation des gains de poids et de l'efficacité alimentaire des zébus et des moutons (Garcia et al., 1973).

En 1977, des feuilles de bananier ont été incorporées dans l'alimentation du zébu nourri à la canne à sucre en République dominicaine. Les résultats obtenus ont été très encourageants (Meyreles and Preston, 1977). En association avec la paille de blé $(75 \%)$ ensilées avec de la mélasse (25\%) et l'urée, les feuilles $\mathrm{du}$ bananier pourraient remplacer pour $50 \%$, le maïs vert dans l'alimentation des vaches, sans altérer le rendement en lait (Balotch et al., 1988).

Il existe de nombreuses expériences indiquant les effets positifs des feuilles de banane dans l'alimentation des bovins de boucherie. En effet, elles pourraient être incorporées jusqu'à 15\% (de matière sèche) dans les rations des vaches en lactation sans altérer significativement la production de lait. Les performances laitières et la digestibilité diminuent au-delà de ce taux, indique ElGhani (1999), en Egypte. Ces résultats sont similaires à ceux de Khattab et al. (2000). Ils ont introduit $15 \%$ de matière sèche de feuilles de banane ensilées avec de la litière de poulets de chair $(40 \%)$ et de la mélasse ou de lactosérum (60\%) dans l'alimentation des buffles en lactation. Les feuilles de bananier ont été bien acceptées, sans aucun effet négatif sur la production de lait. Mais chez les agneaux, la capacité des feuilles de bananier à favoriser leur croissance est limitée, ce qui est probablement dû à un faible apport énergétique (Marie-Magdeleine et al., 2010). C'est l'une des raisons pour lesquelles Katougole et al. (2008) et Mohapatra (2010) suggèrent l'association des feuilles du bananier avec d'autres aliments riches en protéines pour couvrir les besoins nutritionnels des ruminants.

Des lapins ont été nourris avec des régimes contenant soit des feuilles de bananier séchées (30\%), soit des feuilles de bananier fraîches (30\%) soit une combinaison des feuilles fraîches $(15 \%)$ et sèches $(15 \%)$. Ils ont réalisé des gains de poids similaires à ceux 
de leurs congénères consommant des aliments standards du commerce. Toutefois, l'alimentation avec les feuilles fraîches a été plus bénéfique pour les animaux et le rendement net sur les coûts d'alimentation a été plus élevé pour les lapins nourris avec les feuilles séchées (Fomunyam, 1985). Les lapins peuvent être alimentés avec $40 \%$ des feuilles de bananier dans leur régime. Cela est sans effets néfastes sur leur croissance, leur prise alimentaire et leur physiologie. $\mathrm{Au}$ contraire, un taux d'inclusion de $60 \%$ de ces feuilles réduit la prise de matière sèche (Rohilla et al., 2000).

Par ailleurs, les feuilles de bananier (Musa guninata, Musa balbisiana, Musa sapientum) ont été recommandées par Ekwe et al. (2011) aux agriculteurs du Nigéria pour l'alimentation animale. En effet, l'incorporation de feuilles de bananier dans l'alimentation des lapins sevrés, comme fourrage de base a permis d'améliorer leur croissance et de baisser les coûts de production de cette viande (Ekwe et al., 2011).

Les feuilles séchées de plantain, introduites à $10 \%$ dans le régime standard du poulet, n'ont pas affecté l'efficacité alimentaire ou la conversion alimentaire (Marin et al., 2003). Mais dans un régime pour la volaille, les feuilles de bananiers ne peuvent remplacer la farine de poisson, au risque d'affecter négativement les performances des poulets (Islam et al., 1994).

La farine de feuilles de banane pourrait être incorporée jusqu'à $15 \%$ dans le régime des porcs en croissance (García et al., 1991). Ce taux ne peut être augmenté dans le régime car la farine de feuilles de banane a un effet néfaste sur la digestibilité iléale (Ly et al., 1997).

\section{Feuilles, pseudo-troncs et fruit (banane) du bananier}

Les feuilles et les pseudo-troncs du bananier ainsi que la banane ont été introduits en association dans l'alimentation des ruminants (cheval, chèvres, mouton, veaux, agneaux) et du porc.

Aux Seychelles, les jeunes animaux Jersey croisés, nourris avec des feuilles de bananier (Musa paradisiaca) et des pseudotroncs hachés, complétés avec de la mélasse de leucaena ont connu une croissance de 0,5 g / jour. Des pseudo-troncs séchés de banane (Musa cavendishi) ont été incorporées sans risque à $20 \%$ et $50 \%$ (de matière sèche) respectivement dans les régimes alimentaires des chèvres et des moutons (Poyyamozhi et Kadirvel, 1986). Cependant, leurs gains de poids quotidiens ont été faibles. La valeur nutritive des pseudo-troncs séchées de banane est comparable à celle des pailles de céréales communes et autres résidus de récolte tels que la paille de riz ou de canne à sucre (Viswanathan et al., 1989).

Les pseudo-troncs et les fruits du bananier plantain hachés, frais ou ensilés peuvent servir à l'alimentation des bovins et des porcs (Sharrock, 1997).

Chez les veaux, un mélangede pseudotroncs de banane $(40 \%)$ et d'ensilage de maïs $(60 \%)$ accroît de digestibilité et réduit les coûts d'alimentation, mais l'ingéré alimentaire et le gain de poids corporel diminuent (Dormond and Leng, 2001).

En Guadeloupe, une étude a été réalisée pour évaluer la banane (Musa paradisiaca) comme fourrage (feuilles et pseudo-troncs) pour l'alimentation des agneaux, dans le but de tester son impact sur la qualité de la carcasse. Il s'est révélé que l'utilisation des feuilles et des pseudo-troncs de banane n'a eu aucun effet significatif sur les rendements des carcasses des agneaux. A partir de ces premiers résultats, les feuilles de banane et les pseudo-troncs de bananier pourraient être recommandés comme sources de fourrages (Marie-Magdeleine et al., 2009). Les résidus de banane et les feuillages du bananier peuvent être un aliment de base important pour les porcs pour les petits exploitants des zones de production de bananes (Buragohain et al., 2010). 
Tableau 1 : Compositions chimiques de la pulpe et de l'épluchure (peau) de la banane douce.

\begin{tabular}{lcc}
\hline Constituants & Pulpe & Epluchure (peau) \\
\hline Eau $(\%)$ & $73,8 \pm 0,50$ & 83,50 \\
B-carotène $(\mu \mathrm{g} / 100 \mathrm{~g})$ & $55,68 \pm 14,10$ & \\
Vitamine C $(\mathrm{mg} / 100 \mathrm{~g})$ & $4,5 \pm 0,30$ & \\
Protéine $(\%$ de MS) & 2,20 & 1,80 \\
Lipide $\%$ de MS) & 0,10 & 1,70 \\
Amidon $(\%$ de MS) & 10 & 1,20 \\
Glucose $(\%$ de MS) & 5,00 & 2,40 \\
Fructose $(\%$ de MS) & 6,50 & 6,20 \\
Sucrose $(\%$ de MS) & 12 & 2,60 \\
Cellulose $(\%$ de MS) & 9,10 & 8,40 \\
Potassium $(\mathrm{mg} / 100 \mathrm{~g})$ & $318,95 \pm 28,20$ & $78,1 \pm 6,58$ \\
Phosphore $(\mathrm{mg} / 100 \mathrm{~g})$ & $21,7 \pm 2,40$ & $19,2 \pm 0,00$ \\
Calcium $(\mathrm{mg} / 100 \mathrm{~g})$ & $4,9 \pm 1,00$ & $24,3 \pm 0,10$ \\
Sodium $(\mathrm{mg} / 100 \mathrm{~g})$ & $17,35 \pm 3,68$ & \\
Magnésium $(\mathrm{mg} / 100 \mathrm{~g})$ & $30,8 \pm 4,40$ &
\end{tabular}

Tableau 2 : Compositions chimiques des produits et sous- produits du bananier plantain.

\begin{tabular}{lccc}
\hline $\begin{array}{l}\text { Produits et sous- produits du } \\
\text { bananier plantain }\end{array}$ & Fibres brutes & Cendres & Protéine brute \\
\hline Tronc mature & 23,70 & 9,70 & 7,60 \\
Tige du plantain & 13,80 & 15,60 & 2,80 \\
feuilles & 28,80 & 9,30 & 17,30 \\
Epluchures & 4,80 & 4,00 & 1,70 \\
Banane ensilee & 25,90 & 16,30 & 5,50 \\
Bananier entier frais & $3,30^{*}$ & $4,80^{*}$ & $4,80^{*}$ \\
\hline
\end{tabular}

$\left(^{*}\right)$ Valeurs en matière fraîche; Central and South East Asia Tables of Feed Composition (1982).

\section{CONCLUSION}

Les études révèlent, au niveau de la composition chimique, que les feuilles du bananier sont riches en eau (85\%) et pauvre en protéine (10-17\%). Son pseudo-tronc contient également beaucoup d'eau (92-95\%) et de fibres avec une faible teneur en protéine $(2,8-7,6 \%)$. Quant à la pulpe de banane, mise à part l'eau $(73,8 \%)$, elle est essentiellement constituée d'amidon, de sucres, de cellulose, de minéraux majeurs $(\mathrm{K}, \mathrm{P}, \mathrm{Ca}$ et $\mathrm{Na})$ et de quelques vitamines. Les épluchures de banane, dépourvues de vitamines, possèdent les mêmes nutriments que la pulpe, mais à des taux plus bas; car elles sont plus gorgées d'eau $(83,5 \%)$. En somme toutes ces matières sont des sources énergétiques et minérales utiles pour nourrir les animaux.

Utilisés dans l'alimentation des ruminants, du porc, du lapin et de la volaille, les feuilles du bananier, son pseudo-tronc, la banane et ses déchets, ensilés, farineux ou hachés, ont montré leur capacité à favoriser la croissance, à améliorer le rendement carcasse et à maintenir la production laitière. En outre, les produits et sous-produits du bananier ont 
une bonne appétibilité avec des digestibilités et des coefficients d'efficacité élevés. Ils peuvent remplacer les céréales employées dans l'élevage, à des proportions variables $(7,5 \%-75 \%)$ selon l'espèce et le stade physiologique des animaux. $\mathrm{Au}$ plan économique, l'introduction des feuilles du bananier, de son pseudo-tronc, de la banane et de ses déchets dans l'alimentation animale peut contribuer à réduire les coûts de production.

\section{REFERENCES}

Abiliza EK, Muya HMH. 1991. Rumen degradation of dry matter and organic matter of different parts of the banana plant. Livestock Research for Rural Development, 26: 318-325.

Adéniji TA, Barimalaa IS, Tenkouano A, Sani LO, Hart AD. 2008. Antinutrients and heavy metals in new Nigeria Musa hybrid peels with emphasis on utilization in livestock production. Fruits, 63: 65-73.

Adisa VA, Okey EN. 1987. Carbohydrate and protein composition of banana pulp and peel as influenced by ripening and mold contamination. Food Chemistry, 25: 8591.

Ajasin OF, Omole AJ, Oluokun JA, Obi OO, Owosibo A. 2006. Performance Characteristics of weaned rabbit fed plantain peel as replacement for maize. World Journal of Zoology, 1: 30-32.

Baloch GM, Soomro FM, Isani GB, Carpenter JR. 1988. Utilization of banana plant silage as a source of roughage for dairy cows. Journal of Dairy Science, 71: 132.

Bouafou KGM. 2007. Etude de la production d'asticots à partir d'ordures ménagères et de la valeur nutritionnelle de la farine d'asticots séchés (FAS) chez le rat en croissance. Thèse de doctorat, Université de Cocody, Abidjan, 145p.

Buragohain R, Kalita G, Sarma K. 2010. Nutritional significance of banana as swine feed. Indian Veterinary Journal, 87: 301-302.
Clavijo H, Maner JH. 1975. Banano maduro en dietas para cerdos en lactancia. Alpa Memento, 6: 147.

Central and South East Asia. 1982. Tables of Feed Composition, Harris et al., (eds). INFIC Press: 230-235.

Cerning-Beroard J, Le Dividich J. 1976. Valeur alimentaire de quelques produits amylacés d'origine tropicale: étude in vitro et in vivo de la patate douce, de l'igname, du malanga, du fruit à pain et de la banane. Annales de Zootechnie, 25: 155-168.

Cordeiro N, Belgacem MN, Torres IC Moura JCVP. 2004. Chemical composition and pulping of banana pseudo sterms. Industrial Crops and Products, 19: 147154.

Dormond H, Boschini C, Rojas A. 1998. Effect of two levels of ripe banana peel on milk production by dairy cattle. Agronomia Costarricense, 22: 43-49.

Dormond H, Rojas A, Jimenez C, Quiros G. 2001. Effect of increasing levels of bluggoe banana pseudostems added to corn silage as roughage, on Jersey calves growing in confinement, during the dry season. Agronomia Costarricense, 24: 3140.

Ekwe, OO, Osakwe II, Nweze BO. 2011. The effect of replacing maize with cassava "sievate" using banana leaves as basal forage in the diet of weaned rabbit. Ozean Journal of Applied Sciences, 4: 51-58.

El-Ghani AAA. 1999. Utilization of banana plant wastes by lactating Friesian cows. Egyptian Journal of Nutrition and Feeds, 2: 29-37.

Emaga TH, Robert C, Ronkart SN, Wathelet B, Paquot M. 2008. Dietary components and pectin chemical features of peels during ripening in banana and plantain varieties. Biores. Technol., 99: 43464354.

Ffoulkes D, Espejo S, Marie D, Delpeche M, Preston TR. 1977. The banana plant as cattle feed: composition and biomass 
production. Tropical Animal Health and Production, 3: 45-50.

Ffoulkes D, Preston TR. 1978. The banana plant as cattle feed: digestibility and voluntary intake of different proportions of leaf and pseudostem. Tropical Animal Health and Production, 3: 114-117.

Fomunyam RT. 1985. Cabbage and banana/plantain leaf in rabbit diets. Science and Technology Review, 1: 1319.

García CE, Chicco CF, Carnevali AA. 1973. Meal of banana leaves in the feeding of ruminants. Agronomia Tropical Venezuela, 23: 293-299.

Garcia A, Ly J, Dominguez OL. 1991. Uso de diferenes niveles de harina de residuos foliares del platano (Musa spp) en piensos secos para cerdos en preceba resumenes IV Congreso ALVEC, La Habana, 94.

Gidenne T. 1986. Effect of feeding banana in addition to a concentrated diet on the digestion in growing rabbits. Cuniscience, 3: $1-6$.

Heuzé V, Tran G, Archimède H, 2011. Banana Leaves and Pseudostems. INRA, CIRAD, AFZ; 100-120.

Islam MA, Hossain MD, Balbul SM, Howlider MAR. 1994. Unconventional feeds for broilers. Indian Veterinary Journal, 71: 775-780.

Katongole CB, Bareeba FB, Sabiiti EN, Ledin I. 2008. Tutritional characterization of some tropical urban market crops wastes. Animal Feed Science and Technology, 141: 275-291.

Khattab HM, Kholif AM, El-Alamy HA, Salem FA, El-Shewy AA. 2000. Ensiled banana wastes with molasses or whey for lactating buffaloes during early lactation. Asian-Australasian Journal of Animal Science, 13: 619-624.

Le Dividich J, Canope I. 1972. Utilisation des Déchets de Banane dans l'Alimentation $d u$ Porc en Croissance. Journées de recherche porcine en France, INRA-ITP: Paris; 499-500.
Le Dividich J, Canope I. 1974. Valeur alimentaire de la farine de banane et de manioc dans le régime du porcelet sevré à 5 semaines : influence du taux de protéine de la ration. Annales de Zootechnie, 23: 161-169.

Le Dividich J, Sève B, Gepffroy F. 1976. Préparation et utilisation de l'ensilage de banane en alimentation animale : Technique de l'ensilage, composition chimique et bilans des matières nutritives. Annales de Zootechnie, 25: 313-323.

Lustre AO, Sorano MS, Morga NS, Balagot AH, Tunac MM. 1976. Physico-chemical changes in «saba » banana during normal and acetylene-induced ripening. Food Chemistry, 1: 125-137.

Ly J, Garcia A, Dominguez PL. 1997. Chemical composition of plantain foliage (Musa paradisiaca) and the effect of its inclusion in the diet on nutrient digestibility in pig. Journal of Animal and Feed Sciences, 6: 257-267.

Marie-Magdeleine C, Liméa L, Etienne T, Lallo $\mathrm{CH}$, Archimède $\mathrm{H}$, Alexandre $\mathrm{G}$. 2009. The effects of replacing Dichantium hay with banana (Musa paradisiaca) leaves and pseudo-stem on carcass traits of Ovin Martinik sheep. Tropical Animal Health and Production, 41: 1531-1538.

Marie-Magdeleine C, Boval M, Philibert L, Borde A, Archimède H. 2010. Effect of banana foliage (Musa x paradisiaca) on nutrition parasite infection and growth of lambs. Livestock Science, 131: 234-239.

Marin A, Carias D, Maria Cioccia A, Hevia P. 2003. Nutritive value of leaves of Musa paradisiaca and Clitoria ternatea as dilutents in diets for broilers. Interciencia, 28: 51-56.

Meyreles L, Preston TR. 1977. The banana plant as cattle feed: effect of different levels of banana leaves on voluntary intake of-chopped sugar cane stalk. Tropical Animal Health and Production, 3: 229-233. 
Mohapatra D, Mishra S, Sutar N. 2010. Banana and its by-product utilization: an overview. Journal of Scientific and Industrial Research, 69: 323-329.

Ogunsipe MH, Agbede JO. 2010. The replacement value of unripe plantain peels on the growth performance, carcass characteristics and cost implications of rabbit production in the tropical region. Researcher, 2: 24-29.

Oliviera L, Cordeiro N, Evtuguin DV, Torres IC, Sylvestre AJD. 2007. Chemical composition of different morphological parts from «Dwarf Cavendish» banana plant and their potential as non-wood renewable source of natural products. Indian Crops Production, 26: 163-172.

Omole AJ, Ajasin FO, Oluokun JA, Obi OO. 2008. Performance characteristics of weaned rabbit fed plantain peel as remplacement for maize. Nutrition and Food Science, 38: 559-563.

Onibon VO, Abulude FO, Lawal LO. 2007. Nutritional and anti-nutritional composition of some Nigerian fruits. Medwell Journals. Journal of Food Technology, 5: 120-122.

Poyyamozhi VS, Kadirvel R. 1986. The value of banana stalk as a feed for goats. Animal Feed Science and Technology, 15: 95-100.

Preston TR, Leng RA. 1987. Matching Ruminant Production Systems with Available Resources in the Tropics and Subtropics. Armidale, NSW, Penambul Books; 120-126.

Rohilla PP, Bujarbaruah KM. 2000. Effect of banana leaves feeding on growth of rabbits. Indian Veterinary Journal, 72: 902-903.

Ruales J, Polit P, Nair BM. 1990. Evalu ation of the nutritional quality of flakes made of banana pulp and full-fat soya flour. Food Chemistry, 36: 31-43.

Sève B, Le Dividich J, Canope I. 1972. Utilisation des Déchets de Banane "Poyo » par le Porc en Croissance aux Antilles Françaises. Journées de recherche porcine en France, INRA-ITP: Paris; 621.

Sève B, Le Dividich J, Canope I. 1976. Préparation et utilisation de l'ensilage de banane en alimentation animale: Incorporation dans la ration du porc en croissance-finition. Annales de Zootechnie, 25: 325-335.

Sharrock S. 1997. Uses of Musa, édn. Inibap. Montpellier, annual repport, Focus paper III, 42-44.

Tewe OO. 1983. Replacing maize with plantain peels in diets for broilers. Nutrition Reports International, 28: 2329.

Thompson AK. 1995. Bananas and Plantains: Banana Processing, Gowen S (ed.). Chapman and Hall: London; 481-492.

Shoukry MM, El-Bedawy TM, Gihad EA, Ali HM, Salman FM, El-Kady RI, 1999. Utilization of banana wastes as hay and silage by sheep and goats. Egyptian Journal of Nutrition and Feeds, 2: 199221.

Viswanathan K, Kadirvel R, Chandrasekaran D. 1989. Nutritive value of banana stalk (Musa cavendishi) as a feed for sheep. Animal Feed Science and Technology, 22: 327-332.

Wall MM. 2006. Ascorbic acid, vitamin A and mineral composition of banana (Musa sapientum) and papaya (Carica papaya) cultivars grown in Haiwaii. Journal of Food Composition and Analysis, 19 : 434-445. 